\title{
The Hippo transducer TAZ as a biomarker of pathological complete response in HER2-positive breast cancer patients treated with trastuzumab-based neoadjuvant therapy
}

\author{
Patrizia Vici ${ }^{1, *}$, Marcella Mottolese ${ }^{2, *}$, Laura Pizzuti ${ }^{1}$, Maddalena Barba ${ }^{1,3}$, Francesca \\ Sperati ${ }^{4}$, Irene Terrenato ${ }^{4}$, Anna Di Benedetto ${ }^{2}$, Clara Natoli ${ }^{5}$, Teresa Gamucci ${ }^{6}$, \\ Domenico Angelucci ${ }^{7}$, Maria Teresa Ramieri ${ }^{8}$, Luigi Di Lauro ${ }^{1}$, Domenico Sergi ${ }^{1}$, \\ Monica Bartucci ${ }^{9}$, Rosanna Dattilo ${ }^{10}$, Alfredo Pagliuca ${ }^{10}$, Ruggero De Maria ${ }^{3}$ and \\ Marcello Maugeri-Saccà ${ }^{1,3}$ \\ ${ }^{1}$ Division of Medical Oncology B, Regina Elena National Cancer Institute, Rome, Italy \\ 2 Department of Pathology, Regina Elena National Cancer Institute, Rome, Italy \\ ${ }^{3}$ Scientific Direction, Regina Elena National Cancer Institute, Rome, Italy \\ ${ }^{4}$ Biostatistics-Scientific Direction, Regina Elena National Cancer Institute, Rome, Italy \\ ${ }^{5}$ Department of Experimental and Clinical Sciences, University "G. d'Annunzio", Chieti, Italy \\ ${ }^{6}$ Medical Oncology Unit, ASL Frosinone, Frosinone, Italy \\ 7 Division of Pathology, 'SS. Annunziata' Hospital, Chieti, Italy \\ ${ }^{8}$ Division of Pathology, ASL Frosinone, Frosinone, Italy \\ ${ }^{9}$ Rutgers Cancer Institute of New Jersey, New Brunswick, New Jersey, USA \\ ${ }^{10}$ Department of Hematology, Oncology and Molecular Medicine, Istituto Superiore di Sanità, Rome, Italy \\ * These authors contributed equally to this work \\ Correspondence to: Marcello Maugeri-Saccà, email: maugeri@ifo.it \\ Ruggero De Maria, email: demaria@ifo.it
}

Keywords: Hippo pathway, TAZ, HER2-positive breast cancer, neoadjuvant therapy, pathological complete response

Received: July 31,2014 Accepted: September 07, $2014 \quad$ Published: September 08, 2014

This is an open-access article distributed under the terms of the Creative Commons Attribution License, which permits unrestricted use, distribution, and reproduction in any medium, provided the original author and source are credited.

\section{ABSTRACT}

Activation of the Hippo transducer TAZ is emerging as a novel oncogenic route in breast cancer and it has been associated with breast cancer stem cells. Additionally, TAZ expression has been linked with HER-2 positivity. We investigated the association between TAZ expression and pathological complete response in HER2-positive breast cancer patients treated with trastuzumab-based neoadjuvant therapy.TAZ was assessed in diagnostic core biopsies by immunohistochemistry. To categorize samples with low TAZ and samples with high TAZ we generated a score by combining staining intensity and cellular localization. The pathological complete response rate was $78.6 \%$ in patients with low TAZ tumors and $57.6 \%$ in patients with high TAZ tumors $(p=0.082)$. In HER2-enriched tumors there was no significant association between TAZ and pathological complete response, whereas in the luminal B subtype the pathological complete response rate was $82.4 \%$ in tumors with low TAZ and $44.4 \%$ in tumors with high TAZ $(p=0.035)$. This association remained statistically significant when restricting our analysis to triple-positive tumors with expression of both estrogen receptor and progesterone receptor $\geq 50 \%(p=0.035)$. Results from this exploratory study suggest that the TAZ score efficiently predicts pathological complete response in Luminal B, HER2-positive breast cancer patients who received neoadjuvant chemotherapy and trastuzumab. 


\section{INTRODUCTION}

The Hippo pathway is an evolutionarily conserved regulator of tissue growth [1]. Mutations in Hippo pathway components give rise to tissue overgrowth in flies [2-3], and pathway defects have been associated with tumorigenesis in mice [4]. In human cancer mutations in core genes have rarely been detected in targeted and whole-genome sequencing studies [1]. Nevertheless, altered expression of different effectors has been found in a wide variety of tumors [5], thus suggesting that disruption of the Hippo signaling might result from the crosstalk with other perturbed molecular networks. The main function of Hippo pathway consists in negatively regulating two homologous oncoproteins: the transcriptional co-activator with PDZ-binding motif (TAZ) and Yes-associated protein (YAP). Attenuated Hippo signaling activates TAZ and YAP, which in turn feed a variety of tumor-promoting functions spanning from proliferation and cell survival to epithelial-mesenchymal transition and migration [1]. Moreover, Hippo-independent YAP/TAZ activation has been described [6].

In breast cancer (BC), TAZ has also been linked to cancer stem cells (CSCs) [7, 8], an uncommon subpopulation of cancer cells characterized by increased resistance to therapy-induced death stimuli [9]. Indeed, it has been demonstrated that TAZ sustains self-renewal and tumor-forming ability of breast CSCs [7]. We have recently strengthened this association by using molecularly characterized xenografts generated with patient-derived CSCs and their differentiated counterparts [8]. In an orthotopic mouse model we described the role of TAZ as a mediator of breast CSC metastatic ability and chemoresistance [8]. Moreover, in a preliminary analysis conducted in the clinical setting we found a statistically significant correlation between TAZ expression and shorter disease-free survival in a consecutive series of $\mathrm{BC}$ patients, and a positive correlation between TAZ and HER2 positivity [8].

The robustness of our preclinical findings, along with promising early clinical data, prompted this study to explore the association between TAZ, evaluated in diagnostic core biopsies, and pathological complete response (pCR) in HER2-positive BC patients treated with trastuzumab-based neoadjuvant therapy.

\section{RESULTS}

Data on demographics, clinical features, therapy administered and treatment outcomes from 61 HER2-positive BC patients treated with neoadjuvant trastuzumab-based therapy in three Italian Cancer Centers were retrieved from our prospectively maintained database and are illustrated in Table 1.

To investigate the relationship between TAZ and pCR we generated a TAZ score that takes into account its activation status, as detailed in the methods section. We observed no association between standard clinicalmolecular factors and the TAZ score (Table 2), neither did

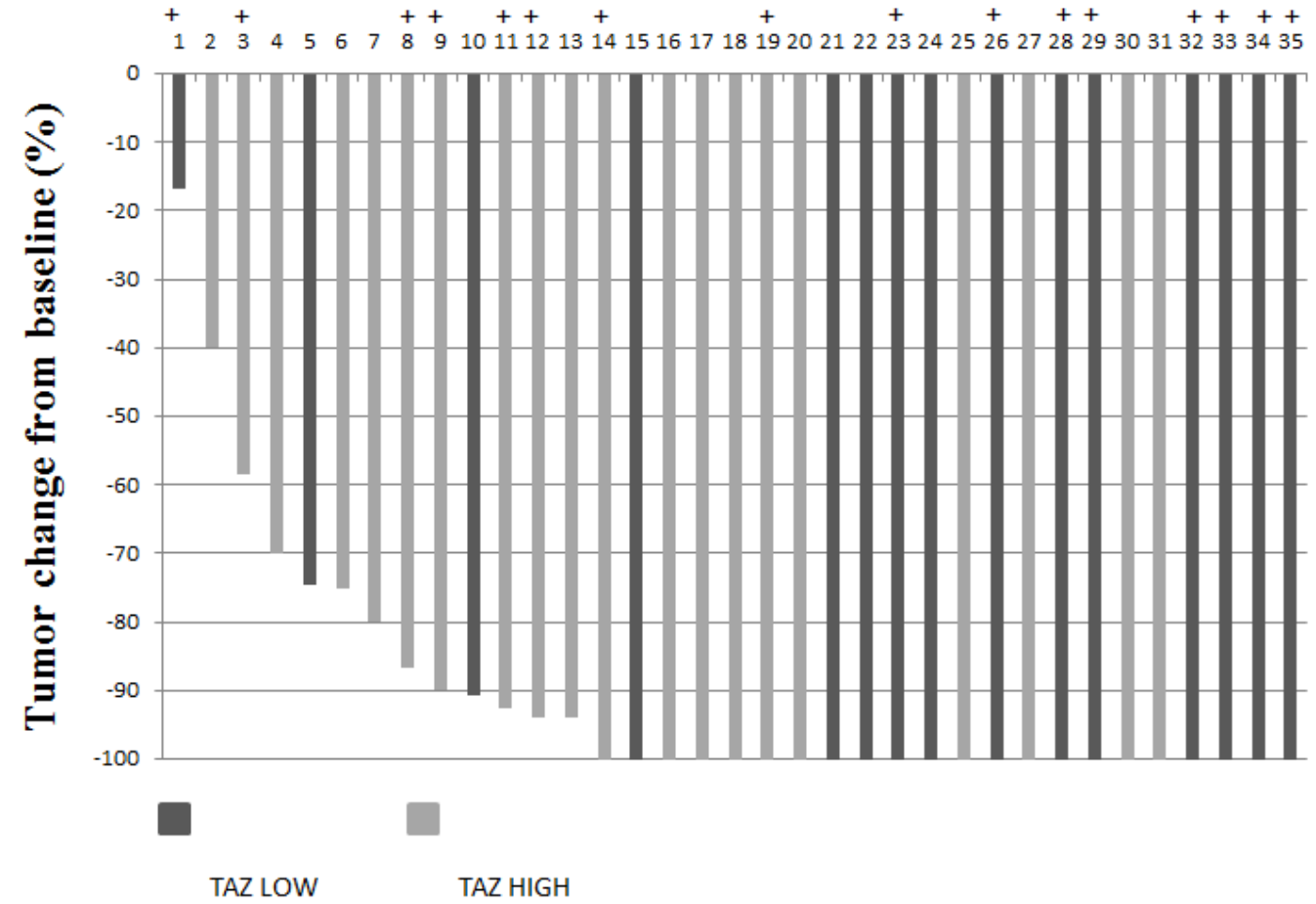

Figure 1: Waterfall plot showing response data for luminal B patients. With + are indicated triple-positive tumors with high expression of hormonal receptors $(\geq 50 \%)$ 
Table 1: Patients' characteristics

\begin{tabular}{|c|c|}
\hline Characteristics & N (\%) \\
\hline $\begin{array}{c}\text { Age at biopsy } \\
\text { Median (range) }\end{array}$ & $48(31-77)$ \\
\hline Clinical stage & $24(39.3)$ \\
II & $37(60.7)$ \\
\hline III & $18(29.5)$ \\
Nodal status & $43(70.5)$ \\
Negative & \\
Positive & $38(62.3)$ \\
\hline Neoadjuvant therapy & $23(37.7)$ \\
EC followed by DT & $34(55.7)$ \\
DT followed by ECT & $27(44.3)$ \\
\hline Menopausal status & \\
Pre & $26(42.6)$ \\
Post & $35(57.4)$ \\
\hline Molecular subtype & \\
HER2-enriched & \\
Luminal B & \\
\hline
\end{tabular}

Abbreviations: E: epirubicin; C: cyclophosphamide; D: docetaxel; T: trastuzumab.

Table 2: Association between clinical-molecular factors and TAZ score

\begin{tabular}{|c|c|c|c|}
\hline Characteristics & $\begin{array}{c}\text { TAZ score } \\
\leq \mathbf{0 . 5 0} \\
\mathrm{N}(\%)\end{array}$ & $\begin{array}{c}\text { TAZ score } \\
>\mathbf{0 . 5 0} \\
\text { N }(\%)\end{array}$ & "p-value \\
\hline Overall population & $28(45.9)$ & $33(54.1)$ & \\
\hline \multicolumn{4}{|l|}{ Clinical stage } \\
\hline II & $9(37.5)$ & $15(62.5)$ & 0.289 \\
\hline III & $19(51.4)$ & $18(48.6)$ & \\
\hline \multicolumn{4}{|l|}{ Nodal status } \\
\hline Negative & $6(33.3)$ & $12(66.7)$ & 0.202 \\
\hline Positive & $22(51.2)$ & $21(48.8)$ & \\
\hline \multicolumn{4}{|l|}{ Neoadjuvant therapy } \\
\hline EC followed by DT & $20(52.6)$ & $18(47.4)$ & 0.175 \\
\hline DT followed by ECT & $8(34.8)$ & $15(65.2)$ & \\
\hline \multicolumn{4}{|l|}{ Menopausal status } \\
\hline Pre & $14(41.2)$ & $20(58.8)$ & 0.406 \\
\hline Post & $14(51.9)$ & $13(48.1)$ & \\
\hline \multicolumn{4}{|l|}{ Molecular subtype } \\
\hline HER2-enriched & $11(42.3)$ & $15(57.7)$ & 0.627 \\
\hline Luminal B & $17(48.6)$ & $18(51.4)$ & \\
\hline
\end{tabular}

Abbreviations: E: epirubicin; C: cyclophosphamide; D: docetaxel; T: trastuzumab. "Chi2 test.

we observe any relationship between standard clinicalmolecular factors and pCR (Table 3).

Overall, forty-one $(67.2 \%)$ patients achieved a pCR (Table 4). In the whole cohort a pCR was recorded in $78.6 \%$ of patients with low TAZ tumors and in $57.6 \%$ of patients with high TAZ tumors, even though this difference was not statistically significant $(p=0.082)$ (Table 4). Neither the TAZ score nor the selected standard molecularclinical features showed evidence of a significant impact on $\mathrm{pCR}$ in the univariate and multivariate logistic regression models (Supplementary Table 1 and 2).

When stratifying by molecular subtype, we did not observe any association between TAZ and pCR in
HER2-enriched tumors (Table 4). In this subtype, the rate of pCRs was equal between cases with low TAZ and cases with high TAZ. Conversely, in the luminal B subtype the pCR rate was $82.4 \%$ in tumors with low TAZ and $44.4 \%$ in tumors with high TAZ ( $\mathrm{p}=0.035$ ) (Table 4$)$. The association was confirmed by the regression model ( $\leq$ 0.50 vs $>0.50 \mathrm{OR}=5.83 ; 95 \% \mathrm{CI}, 1.23-27.63 ; \mathrm{p}=0.026$; supplementary Table 3 ). Finally, a statistically significant association was found in the subset of triple-positive tumors with high expression $(\geq 50 \%)$ of both hormonal receptors $(p=0.035)$ (Table 4$)$. Individual responses in luminal B tumors are illustrated in figure 1. 
Table 3: Association between standard clinical-molecular factors and pCR

\begin{tabular}{|c|c|c|c|}
\hline Characteristics & $\begin{array}{c}\text { No pCR } \\
\text { N (\%) }\end{array}$ & $\begin{array}{c}\text { pCR } \\
\text { N (\%) }\end{array}$ & "p-value \\
\hline Overall population & $20(32.8)$ & $41(67.2)$ & \\
\hline Clinical stage & & & \\
\hline II & $6(25.0)$ & $18(75.0)$ & 0.297 \\
\hline III & $14(37.8)$ & $23(62.2)$ & \\
\hline Nodal status & & & 0.511 \\
\hline Negative & $7(38.9)$ & $11(61.1)$ & \\
\hline Positive & $13(30.2)$ & $30(69.8)$ & \\
\hline Neoadjuvant therapy & & & \\
\hline EC followed by DT & $11(28.9)$ & $27(71.1)$ & \\
\hline DT followed by ECT & $9(39.1)$ & $14(60.9)$ & \\
\hline Menopausal status & & & \\
\hline Pre & $11(32.4)$ & $23(67.6)$ & 0.935 \\
\hline Post & $9(33.3)$ & $18(66.7)$ & \\
\hline Molecular subtype & & & \\
\hline HER2-enriched & $7(26.9)$ & $19(73.1)$ & \\
\hline Luminal B & $13(37.1)$ & $22(62.9)$ & \\
\hline
\end{tabular}

Abbreviations: pCR: pathological complete response; E: epirubicin; C: cyclophosphamide; D: docetaxel; T: trastuzumab. "Chi2 test.

Table 4: Association between the TAZ score and pCR

\begin{tabular}{|c|c|c|c|c|}
\hline & & $\begin{array}{c}\text { No pCR } \\
\text { N }(\%)\end{array}$ & $\begin{array}{c}\text { pCR } \\
\mathrm{N}(\%)\end{array}$ & p-value \\
\hline \multirow[t]{2}{*}{ Overall population } & & $20(32.8)$ & $41(67.2)$ & \\
\hline & $\begin{array}{c}\text { TAZ score } \\
\leq 0.50 \\
>0.50\end{array}$ & $\begin{array}{r}6(21.4) \\
14(42.4)\end{array}$ & $\begin{array}{l}22(78.6) \\
19(57.6)\end{array}$ & $=0.082$ \\
\hline \multirow[t]{2}{*}{ HER2-enriched } & & $7(26.9)$ & $19(73.1)$ & \\
\hline & $\begin{array}{c}\text { TAZ score } \\
\leq 0.50 \\
>0.50\end{array}$ & $\begin{array}{l}3(27.3) \\
4(26.7)\end{array}$ & $\begin{array}{r}8(72.7) \\
11(73.3)\end{array}$ & ${ }^{\circ} 0.035$ \\
\hline \multirow[t]{2}{*}{ Luminal B } & & $13(37.1)$ & $22(62.9)$ & \\
\hline & $\begin{array}{c}\text { TAZ score } \\
\leq 0.50 \\
>0.50\end{array}$ & $\begin{array}{c}3(17.6) \\
10(55.6)\end{array}$ & $\begin{array}{c}14(82.4) \\
8(44.4)\end{array}$ & ${ }^{\circ} 0.03$ \\
\hline \multirow[t]{2}{*}{ TP50 } & & $6(37.5)$ & $10(62.5)$ & \\
\hline & $\begin{array}{c}\text { TAZ score } \\
\leq 0.50 \\
>0.50\end{array}$ & $\begin{array}{l}1(11.1) \\
5(71.4)\end{array}$ & $\begin{array}{l}8(88.9) \\
2(28.6)\end{array}$ & ${ }^{\circ} 0.03$ \\
\hline
\end{tabular}

Abbreviations: pCR: pathological complete response; TP50: triple-positive tumors with expression of estrogen receptor and progesterone receptor $\geq 50 \%$. "Chi2 test, ${ }^{\circ}$ Fisher Exact test.

\section{DISCUSSION}

In this study we evaluated whether an association existed between TAZ and pCR in 61 HER2-positive BC patients who received neoadjuvant chemotherapy plus trastuzumab. To our knowledge, this is the first study investigating the predictive role of the Hippo transducer TAZ in this setting. No statistically significant association between TAZ and pCR was reported in the entire cohort. However, an association emerged when stratifying by 
molecular subtype. While TAZ did not have any impact on pCR in HER2-enriched tumors, a significant association was observed in Luminal B tumors and in the subset with high expression of both hormonal receptors. As we did not note any association between standard clinical-molecular features and pCR, we provided hints supporting the hypothesis that TAZ is an independent predictive factor for $\mathrm{pCR}$. Indeed, the inclusion of the proliferation marker $\mathrm{Ki}-67$ in the models did not affect our study results (data available upon request).

In interpreting our results, we are aware that this study is limited by its retrospective nature and the relatively restricted number of patients evaluated. Nevertheless, our study has some important strengths. First, the experimental path underlying this study deserves to be mentioned. Patient-derived xenografts generated with CSCs allow to reconstitute human tumors in the murine background and to amplify them through serial transplantation $[10,11]$. As mentioned above, our interest on TAZ stemmed from molecularly characterized patient-derived xenografts obtained with the delivery of mammospheres, which are enriched for CSCs, generated from patients with Luminal B tumors [8]. Thus, our preclinical model may represent a promising tool for streamlining the preclinical-clinical transition of novel biomarkers.

Second, over the past decade many prognostic and predictive multigene classifiers have been developed to assist in clinical decision-making [12]. Notwithstanding, even the broadest investigated genomic assays originally developed to foresee long-term outcomes are struggling to gain widespread consensus due to the unclear gain in precision and cost-effectiveness over standard prognostic factors [13]. We developed a clinically applicable assay with the potential to expand the current pipeline of biomarkers used in routine clinical practice. From a biological standpoint, it is worth mentioning that the TAZ score employed in this study was generated by considering cellular localization, and consequently TAZ activation. Indeed, cytoplasmic accumulation reflects ubiquitinmediated proteolysis, whereas nuclear translocation is usually interpreted as an activation of the TAZ-mediated oncogenic transcriptional program [14].

As pCR in an established surrogate marker for better long-term outcomes [15], it is of utmost importance to identify biomarkers of response and resistance to HER2-directed therapy. Even though a variety of putative predictive biomarkers have been proposed, spanning from gene expression profiles to mutations in HER2 and genes belonging to its transduction machinery [16-19], we are still waiting for validation studies. Even considering the sample size of our study, the magnitude of the difference in the pCR rate seen in Luminal B tumors according to TAZ status is rather uncommonly reported, and in our opinion represents a background for a prospective study with biomarker validation purposes. Moreover, the lesson we learned from clinical trials, such as NeoALTTO [20], GEPARQUINTO [21], and NeoSphere [22], is that hormone receptor-positive tumors achieve a lower rate of pCR compared with hormone receptor-negative tumors. Thus, foreseeing pCR after anti-HER2-based neoadjuvant therapy acquires an even more relevant therapeutic implication in this setting. With the present analysis we have provided clues on the potential predictive value of a TAZ-based biomarker in HER2-positive and hormone receptor-positive tumors.

In summary, the message conveyed by this study is that the TAZ score we developed appears extremely promising in predicting the individual likelihood to achieve a pCR to trastuzumab-based neoadjuvant therapy in Luminal B, HER2-positive BC patients. As a consequence, we plan to prospectively validate the TAZ score as a molecular predictor in this BC subtype. Finally, our goal is to examine core components of the Hippo pathway in different therapeutic settings and molecular subtypes with the aim of identifying Hippo pathwayrelated prognostic and predictive biomarkers.

\section{METHODS}

\section{Study Participants and procedures}

Included patients were 61 HER2-positive BC patients who received neoadjuvant trastuzumab-based therapy. Patients were considered suitable for inclusion if they had received trastuzumab as a part of their neoadjuvant treatment, had complete data on baseline clinical features, therapy administered and treatment outcomes, if the planned treatment was completed, and the amount of biological materials was sufficient for TAZ analysis in their biopsies. Patients were treated with two different schedules: epirubicin $120 \mathrm{mg} / \mathrm{m}^{2}$ plus cyclophosphamide $600 \mathrm{mg} / \mathrm{m}^{2}$ administered intravenously (IV) on day 1 every 21 days for four cycles followed by docetaxel $100 \mathrm{mg} / \mathrm{m}^{2}$ plus trastuzumab $6 \mathrm{mg} / \mathrm{kg}$ (after a loading dose of $8 \mathrm{mg} / \mathrm{kg}$ ) administered IV on day 1 every 3 weeks for four cycles, or a reverse sequence with the administration of trastuzumab for the whole length of chemotherapy. pCR was defined as no residual invasive tumor in both breast and axilla (ypT0/is ypN0), and was assessed by local pathologists. The immunohistochemical assessment of estrogen receptor (ER), progesterone receptor $(\mathrm{PgR})$ and HER2 was performed in formalinfixed paraffin-embedded tissues using the monoclonal antibodies (MoAbs) 6F11, 1A6 (Menarini, Florence, Italy) and the polyclonal antibody A0485 (PoAb, Dako, Milan, Italy). TAZ was evaluated with the MoAb anti-TAZ (M2616, BD Pharmingen, San Jose, CA,USA). ER and PgR were considered positive when $\geq 1 \%$ of the neoplastic cells showed a distinct nuclear immunoreactivity. 
HER2 positivity was defined, according to ASCO-CAP guidelines, as 3+ overexpression by immunohistochemical testing or as 2+ with HER2 amplification by silver in situ hybridization (SISH, Inform HER2 DNA Probe; Inform Chr17 probe, Roche Diagnostics, Milan, Italy) [23]. TAZ was evaluated in diagnostic core biopsies and considered positive when $>10 \%$ of tumor cells displayed nuclear and/ or cytoplasmic immunostaining. TAZ staining intensity was graded on a four-grade scale ( 0 : negative, $1+$ : weak, $2+$ : moderate, $3+$ : strong). The TAZ score was obtained by multiplying the staining intensity $\mathrm{x} 1.5$ (nuclear localization) or 0.5 (cytoplasmic localization), in order to take into account its activation status. Using the median score of all tumors as a cut-off point, tumors with a score $\leq 0.5$ were considered as TAZ low, whereas tumors with a score $>0.5$ as TAZ high. TAZ expression and localization were independently evaluated by two investigators (MM and ADB) who were masked to treatment outcome. Discordant cases were reviewed at a face-to-face meeting.

\section{Statistical analysis}

We computed descriptive statistics for all variables of interest. Continuous data were reported as mean and standard deviation and we represented categorical data with frequencies and percentage values. In order to assess the relationships between categorical variables, different tests were employed: the Pearson's Chi-squared test of independence (2-tailed) and the Fisher Exact test. We used a univariate logistic regression model to identify variables that could influence the study outcome (pCR). We planned to insert variables significant in the univariate analysis in a multivariate logistic regression. We considered statistically significant $\mathrm{p}$ values less than 0.05 . Statistical analyses were carried out using SPSS software (SPSS version 21, SPSS Inc., Chicago, IL, USA).

\section{ACKNOWLEDGEMENTS}

We thank Tania Merlino and Ana Maria Edlisca for technical assistance. Funding: This study was supported by Italian Association for Cancer Research (AIRC) Investigator Grant to RDM.

\section{REFERENCES}

1. Harvey KF, Zhang X, Thomas DM. The Hippo pathway and human cancer. Nat Rev Cancer. 2013;13:246-257.

2. Tapon N, Harvey KF, Bell DW, Wahrer DC, Schiripo TA, Haber D, Hariharan IK. Salvador Promotes both cell cycle exit and apoptosis in Drosophila and is mutated in human cancer cell lines. Cell. 2002;110:467-478.

3. Justice RW, Zilian O, Woods DF, Noll M, Bryant PJ. The Drosophila tumor suppressor gene warts encodes a homolog of human myotonic dystrophy kinase and is required for the control of cell shape and proliferation. Genes Dev.;9:534546.

4. Camargo FD, Gokhale S, Johnnidis JB, Fu D, Bell GW, Jaenisch R, Brummelkamp TR. YAP1 increases organ size and expands undifferentiated progenitor cells. Curr Biol. 2007; 17:2054-2060.

5. Johnson R, Halder G. The two faces of Hippo: targeting the Hippo pathway for regenerative medicine and cancer treatment. Nat Rev Drug Discov. 2014;13:63-79.

6. Azzolin L, Panciera T, Soligo S, Enzo E, Bicciato S, Dupont S, Bresolin S, Frasson C, Basso G, Guzzardo V, Fassina A, Cordenonsi M, Piccolo S. YAP/TAZ Incorporation in the $\beta$-Catenin Destruction Complex Orchestrates the Wnt Response. Cell. 2014;158:157-170.

7. Cordenonsi M, Zanconato F, Azzolin L, Forcato M, Rosato A, Frasson C, Inui M, Montagner M, Parenti AR, Poletti A, Daidone MG, Dupont S, Basso G, et al. The Hippo transducer TAZ confers cancer stem cell-related traits on breast cancer cells. Cell. 2011;147:759-772.

8. Bartucci M, Dattilo R, Moriconi C, Pagliuca A, Mottolese M, Federici G, Benedetto AD, Todaro M, Stassi G, Sperati F, Amabile MI, Pilozzi E, Patrizii M, et al. TAZ is required for metastatic activity and chemoresistance of breast cancer stem cells. Oncogene. 2014 Feb 17. doi: 10.1038/ onc.2014.5. [Epub ahead of print]

9. Maugeri-Saccà M, Vigneri $\mathrm{P}$, De Maria R. Cancer stem cells and chemosensitivity. Clin Cancer Res. 2011;17:49424947.

10. Siolas D, Hannon GJ. Patient-derived tumor xenografts: transforming clinical samples into mouse models. Cancer Res. 2013;73:5315-5319.

11. Baiocchi M, Biffoni M, Ricci-Vitiani L, Pilozzi E, De Maria R. New models for cancer research: human cancer stem cell xenografts. Curr Opin Pharmacol. 2010;10:380-384.

12. Reis-Filho JS, Pusztai L. Gene expression profiling in breast cancer: classification, prognostication, and prediction. Lancet. 2011;378:1812-1823.

13. NCCN Clinical Practice Guidelines in Oncology: Breast Cancer, Version 3.2014. Available at: http://www.nccn.org/ professionals/physician_gls/f_guidelines.asp

14. Pan D. The hippo signaling pathway in development and cancer. Dev Cell. 2010;19:491-505.

15. Fumagalli D, Bedard PL, Nahleh Z, Michiels S, Sotiriou C, Loi S, Sparano JA, Ellis M, Hylton N, Zujewski JA, Hudis C, Esserman L, Piccart M; BIG-NABCG collaboration. A common language in neoadjuvant breast cancer clinical trials: proposals for standard definitions and endpoints. Lancet Oncol. 2012;13:e240-248.

16. Prat A, Bianchini G, Thomas M, Belousov A, Cheang MC, Koehler A, Gómez P, Semiglazov V, Eiermann W, Tjulandin S, Byakhow M, Bermejo B, Zambetti M, et al. Research-based PAM50 subtype predictor identifies higher responses and improved survival outcomes in HER2positive breast cancer in the NOAH study. Clin Cancer Res. 
2014;20:511-521.

17. Loi S, Michiels S, Lambrechts D, Fumagalli D, Claes B, Kellokumpu-Lehtinen PL, Bono P, Kataja V, Piccart MJ, Joensuu H, Sotiriou C. Somatic mutation profiling and associations with prognosis and trastuzumab benefit in early breast cancer. J Natl Cancer Inst. 2013;105:960-967.

18. Perez EA, Dueck AC, McCullough AE, Chen B, Geiger XJ, Jenkins RB, Lingle WL, Davidson NE, Martino S, Kaufman PA, Kutteh LA, Sledge GW, Harris LN, et al. Impact of PTEN protein expression on benefit from adjuvant trastuzumab in early-stage human epidermal growth factor receptor 2-positive breast cancer in the North Central Cancer Treatment Group N9831 trial. J Clin Oncol. 2013;31:2115-2122.

19. Scaltriti M1, Rojo F, Ocaña A, Anido J, Guzman M, Cortes J, Di Cosimo S, Matias-Guiu X, Ramon y Cajal S, Arribas J, Baselga J. Expression of p95HER2, a truncated form of the HER2 receptor, and response to anti-HER2 therapies in breast cancer. J Natl Cancer Inst. 2007;99:628-638.

20. Baselga J1, Bradbury I, Eidtmann H, Di Cosimo S, de Azambuja E, Aura C, Gómez H, Dinh P, Fauria K, Van Dooren V, Aktan G, Goldhirsch A, Chang TW, et al. Lapatinib with trastuzumab for HER2-positive early breast cancer (NeoALTTO): a randomised, open-label, multicentre, phase 3 trial. Lancet 2012;379:633-640.

21. Untch M1, Loibl S, Bischoff J, Eidtmann H, Kaufmann M, Blohmer JU, Hilfrich J, Strumberg D, Fasching PA, Kreienberg R, Tesch H, Hanusch C, Gerber B, et al. Lapatinib versus trastuzumab in combination with neoadjuvant anthracycline-taxane-based chemotherapy (GeparQuinto, GBG 44): a randomised phase 3 trial. Lancet Oncol. 2012;13:135-144.

22. Gianni L, Pienkowski T, Im YH, Roman L, Tseng LM, Liu MC, Lluch A, Staroslawska E, de la Haba-Rodriguez J, Im SA, Pedrini JL, Poirier B, Morandi P, et al. Efficacy and safety of neoadjuvant pertuzumab and trastuzumab in women with locally advanced, inflammatory, or early HER2-positive breast cancer (NeoSphere): a randomised multicentre, open-label, phase 2 trial. Lancet Oncol. 2012; 13:25-32.

23. Wolff AC, Hammond ME, Hicks DG, Dowsett M, McShane LM, Allison KH, Allred DC, Barlett JM, Bilous M, Fitzgibbons P, Hanna W, Jenkins RB, Mangu PB, et al. Recommendations for Human Epidermal Growth Factor Receptor 2 Testing in Breast Cancer: American Society of Clinical Oncology/College of American Pathologists Clinical Practice Guideline Update. J Clin Oncol. 2013; 31:3997-4013. 